\title{
Improved Accuracy Factors for the Nonuniform Fast Fourier Transform (NUFFT) Algorithm
}

\author{
Jen-Tsai Kuo, Senior Member, IEEE, and Hsin-Ying Lee
}

\begin{abstract}
Based on the regular Fourier matrix, a new set of accuracy factors is proposed for the nonuniform fast Fourier transform algorithm to improve the accuracy of transformed data. It shows that the proposed factors can reduce the errors by three to more than ten times with almost the same number of arithmetic operations. Numerical examples are shown for the applications in computational electromagnetics.
\end{abstract}

Index Terms-Accuracy factor, fast Fourier transform (FFT), microstrip line, nonuniform sampling.

\section{INTRODUCTION}

$\mathbf{T}$ HE fast Fourier transform (FFT) algorithm has played a very important role in developing science and technology since it was proposed [1]. One of its important properties is that the input data must be sampled with equal distance and the transformed results are also uniformly spaced. In many applications, however, if the samples can be nonuniformly spaced, the computation and computer memory can be much more efficient. To achieve this goal, several studies [2]-[4] investigate the FFT algorithm for nonuniformly sampled data. In [2], based on certain analytical considerations, a group of algorithms is presented for generalizing both the forward and backward FFTs for the cases of nonuniform samples and nonequispaced nodes. Later, a new NUFFT algorithm with a comparable computation complexity is developed in [3], [4] and provides results with much more accuracy.

Incorporated into the spectral-domain approach (SDA) for analyzing shielded multiple coupled microstrip lines, the NUFFT algorithm can be 60 times faster than the traditional SDA calculation for results with similar accuracy [5]. The NUFFT is also extended to process 2-D data for SDA analyses of planar circuit discontinuities [6].

The innovation in the new NUFFT algorithm developed in [3] includes the use of the cosine accuracy factors and the least squares solution formulation. It leads to a closed-form solution for the coefficients of the local $q+1$ regular Fourier bases to expand a nongrid point and saves many arithmetic operations.

Manuscript received July 29, 2008; revised September 05, 2008. First published December 22, 2008; current version published January 08, 2009. This work was supported in part by the Ministry of Education under the ATU Program and by the National Science Council of Taiwan, under Grants NSC 95-2221-E009-037-MY2 and NSC 96-2221-E-009-245.

The authors are with the Department of Communication Engineering, National Chiao Tung University, Hsinchu 300, Taiwan (e-mail: jtkuo@ mail.nctu. edu.tw).

Color versions of one or more of the figures in this paper are available online at http://ieeexplore.ieee.org.

Digital Object Identifier 10.1109/LMWC.2008.2008518
In this letter, we study the improvement of the accuracy of the NUFFT algorithm by choosing an alternative set of accuracy factors. The improvement will be shown by comparing the errors with those obtained by the accuracy factors used in [3].

\section{NUFFT ALGORITHM AND ACCURACY FACTORS}

The key step of the NUFFT is to approximate a nonuniform sample in the original domain by interpolating $q+1$ regular Fourier bases, in the neighborhood of the sample, of an oversampled FFT with finite nonzero coefficients. This idea is equivalent to finding a $(q+1) \times 1$ vector $\underline{x}=\left(x_{-q / 2}, \ldots, x_{q / 2}\right)^{t}$ to satisfy the following approximation:

$$
s_{j} \omega^{j m c} \approx \sum_{k=[m c]-q / 2}^{[m c]+q / 2} x_{k-[m c]}(c) \omega^{j k}
$$

where the superscript $t$ denotes the transpose operation, $c$ is an arbitrary real number, $m$ is an integer no less than 2 and known as the oversampling rate, $s_{j}(j=-N / 2, \ldots, N / 2-1)$ are the accuracy factors, $\omega=e^{i 2 \pi / m N}$ is the basis of the regular FFT of size $m N, i=\sqrt{-1}, N$ is number of data points, and $[m c]$ represents the integer closest to $m c$.

The approximation (1) can be written in a matrix form $\underline{v}(c) \approx$ $A x(c)$, which consists of $N$ equations with $q+1$ variables. In [3], the solution to least squares of the error $e=\|\underline{v}(c)-\underline{A x}(c)\|$ is

$$
\underline{x}(c)=\left(\underline{A}^{T} \underline{A}\right)^{-1} \underline{A}^{T} \underline{v}(c)
$$

where the superscript $T$ denotes the conjugate transpose operation. While the entries of $\underline{A}$ depend on $c$, the product matrix $\underline{A}^{T} \underline{A}$ has a closed-form result independent of $c$.

In [3], the data obtained by the cosine factors $\left(s_{j}\right.$ 's) have 12 to 14 times better accuracy than those by the Gaussian factors [2]. This reveals that the accuracy of the NUFFT algorithm does depend on the accuracy factors. We propose the following accuracy factors:

$$
s_{j}(n)=\cos ^{n}\left(\frac{j \pi}{m N}\right)
$$

where $n>0$. The entry of $\underline{A}^{T} \underline{v}(c)$ can then be written as

$$
a_{j n}(c)=\sum_{\ell=-N / 2}^{N / 2-1} \cos ^{n}\left(\frac{\ell \pi}{N m}\right) e^{i \frac{2 \ell \pi}{m N}\left(\{m c\}+\frac{q}{2}-j\right)}
$$

where $\{m c\}=m c-[m c]$. When $n=1,(3)$ is the cosine factors proposed in [3] and the closed-form expression of $a_{j n}(c)$ in (4) can be found in [4]. It is the existence of the closed-form result that saves much CPU time for the NUFFT computation. 
If $a_{j n}(c)$ has no closed-form result, an increase of arithmetic operations becomes inevitable. The increased number of operations is proportional to $N^{2}$ since the direct $N$-term summation has to be applied to each of the $N$ sample points. If $n$ is an integer, however, these extra operations can be saved since a general closed-form expression can be derived as follows. The factors $\cos ^{n} \theta$ in (3) and (4) can be expanded as a series of the cosine functions of multiples of its argument [7]

$$
\begin{aligned}
\cos ^{2 k} \theta & =\frac{1}{2^{2 k}}\left[\sum_{i=0}^{k-1} 2\left(\begin{array}{c}
2 k \\
i
\end{array}\right) \cos 2(k-i) \theta+\left(\begin{array}{c}
2 k \\
k
\end{array}\right)\right] \\
\cos ^{2 k-1} \theta & =\frac{1}{2^{2 k-2}} \sum_{i=0}^{k-1}\left(\begin{array}{c}
2 k-1 \\
i
\end{array}\right) \cos (2 k-2 i-1) \theta .
\end{aligned}
$$

Define

$$
b_{j n}(c)=\sum_{\ell=-N / 2}^{N / 2-1} \cos \frac{\ell n \pi}{N m} e^{i \frac{2 \ell \pi}{m N}\left(\{m c\}+\frac{q}{2}-j\right)} .
$$

Then it can be validated that $b_{j n}(c)=i\left(S_{j n}^{+}+S_{j n}^{-}\right)$, and

$$
S_{j n}^{ \pm}=\frac{\sin \left(\frac{\pi}{2 m}(2 j \mp k-q-2\{m c\})\right)}{1-e^{-i \frac{\pi}{m N}(2 j \mp k-q-2\{m c\})}} .
$$

Thus, when $n$ is odd, we have

$$
a_{j n}(c)=\frac{1}{2^{n-1}} \sum_{\ell=0}^{(n-1) / 2}\left(\begin{array}{l}
n \\
i
\end{array}\right) b_{j, n-2 \ell}(c) .
$$

The closed-form result of $a_{j n}(c)$ for even $n$ can be derived in a similar fashion. With these closed-form expressions, the new NUFFT implementation will take almost the same CPU time as the original $n=1$ case in [3].

\section{NUMERICAL RESULTS}

The current density on a microstrip line shown in Fig. 1 is used to test the accuracy of the NUFFT algorithm. The density function is given as

$$
f(u)=\frac{1}{\sqrt{1-\left(\frac{2 u}{W}\right)^{2}}}
$$

where $W$ is the line width and the function $f$ has singularities at $u= \pm W / 2$. In the NUFFT, the sampled points $y_{\ell}$ are chosen at

$$
y_{\ell}= \begin{cases}\left(\frac{W}{2}-0.005\right) \times \cos \left(\frac{\ell \pi}{2(N / 4-1)}-\frac{\pi}{2}\right), & \left|y_{\ell}\right|<\frac{W}{2} \\ \frac{N}{2}-\left(\frac{N}{2}-\frac{W}{2}-0.005\right) \times \cos \left(\frac{\ell \pi}{N}\right), & \left|y_{\ell}\right|>\frac{W}{2}\end{cases}
$$

The two errors defined in [2], $E_{2}$ and $E_{\infty}$, are used for measuring the accuracy of the NUFFT algorithm. Given $W=2$ and 6, Fig. 2 plots $E_{2}$ and $E_{\infty}$ using $N=100, m=2$ and $q=8$ for $0<n \leq 8$. The curves show that both $E_{2}$ and $E_{\infty}$ are close to being independent of the two line widths, except that around $n=2,4$ and 6 the $E_{\infty}$ values for $W=6$ are a little bit higher than those for $W=2$. The best results occur at $n=4$ where both $E_{2}$ and $E_{\infty}$ are about 0.18 to 0.07 times those at $n=1$.

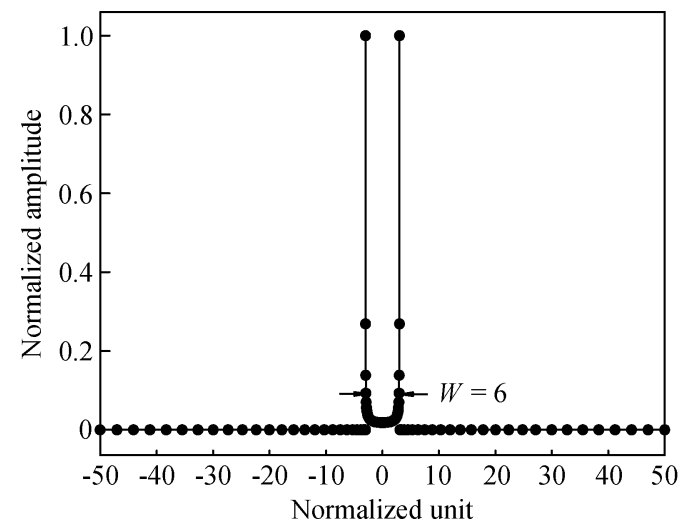

Fig. 1. Current density on a microstrip line and the samples in the NUFFT.

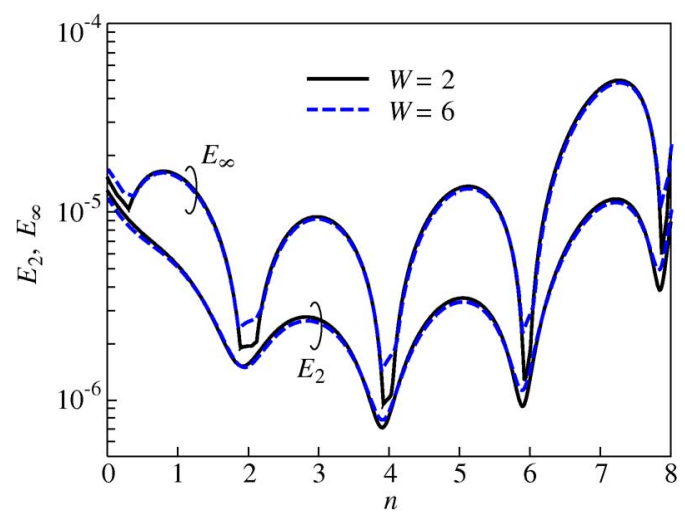

Fig. 2. Comparison of the $E_{2}$ and $E_{\infty}$ errors of the NUFFT results of the currents in Fig. 1. $m=2, q=8, N=100$.

The current density in Fig. 1 is a symmetric wave, so that the Fourier transformed data will be real and symmetric. To check if asymmetric input data degrade the errors, the microstrip line is offset by $M$ units. Fig. 3 compares the $E_{2}$ errors for $M=15,15.05, \ldots, 15.25$. It is found that the curve for $M=$ $15.25+\Delta$ overlaps with that for $M=15.25-\Delta, \Delta=$ $0.05,0.1, \ldots, 0.25$. Also, the six curves are obtained periodically when $M$ is increased or decreased by 0.5 units. The periodicity is obtained when $N$ is changed to 154 and 254. As shown in Fig. 3, when $M$ is increased from 15 to 15.1 , values of $n$ with the minimal $E_{2}$ move quickly from even to close to odd numbers. When $M=15.25$, one can see that the best $E_{2}$ occur at $n=3$ and 5. The $E_{2}$ values at $n=1$ and 7 are about three times larger that at $n=3$. When $M=15.05$, the $E_{2}$ values for both $n=3$ and 4 are about half of that for $n=1$.

The CPU time consumed by the extra arithmetic operations required for a general power $n$ in (3) is investigated as follows. A case study with $N=2^{10}$ nonuniform points is tested for 80 times and the average CPU seconds are listed in Table I. The program is coded for the MATLAB version 6.0 and executed on a uniprocessor PC with the AMD Athlon CPU of $1.83 \mathrm{GHz}$. When $n \neq$ integer, the matrices $\underline{A}(c)$ and $\underline{A}^{T} \underline{v}(c)$ have to be calculated for each sample $c$ for $2^{10}$ times, and they take 4.6 and $1.3 \mathrm{~s}$, respectively. The high set-up cost may make the new accuracy factor non-competitive. Fortunately, it is found that for 


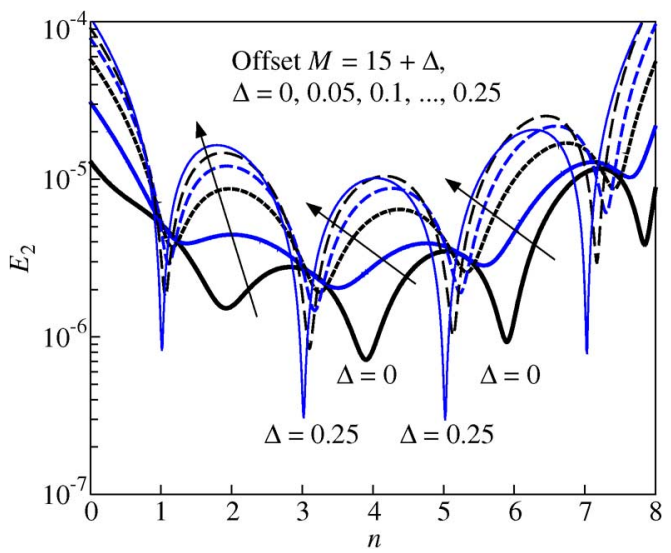

Fig. 3. $E_{2}$ errors for the NUFFT results of currents of offset microstrips.

TABLE I

COMPARISON OF CPU SECONDS USED FOR THE NUFFT ALGORITHM WITH DIFFERENT SETS OF ACCURACY FACTORS

\begin{tabular}{|c|c|c|c|}
\hline Calculation & No. of calls & $(3)(n \neq$ int. $)$ & {$[3](n=1)$} \\
\hline$\underline{A}(c)$ & 1024 & 4.562 & - \\
\hline$\underline{A}^{T} \underline{v}(c)$ & 1024 & 1.283 & 0.015 \\
\hline$(2)$ & 1024 & 0.033 & 0.033 \\
\hline NUFFT $(m N=2048)$ & 1 & 0.078 & 0.078 \\
\hline
\end{tabular}

most of the case studies shown here the minimal $E_{2}$ and $E_{\infty}$ occur at or near $n=$ integer. For these cases, as compared with [3], the extra CPU time is negligible.

It is possible to increase $m$ or $q$ for the original $n=1$ case to enhance its accuracy. Figs. 4 and 5 investigate the dependences of the errors on the parameters $m$ and $q$, respectively, for the symmetric current density in Fig. 1 with $W=2$. In Fig. 4, both $E_{2}$ and $E_{\infty}$ are plotted against $n$ for $m=2,3$ and 4 . Note that based on the $N \log _{2} N$ rule, the CPU time for $m=4$ will be more than two times that for $m=2$. When $m=2,3$ and 4 , the best errors occur around $n=4$ where $E_{2}$ is close to $10^{-6}$, $10^{-8}$ and $10^{-9}$, respectively. Fig. 5 plots the data for $q=8,10$ and 12. When $q=8$, again, the best errors occur at $n=4$ and when $q=12$ the best $n$ is close to 6 . The $E_{2}$ values for $n=4$ and 6 are only 0.25 to 0.06 times those for $n=1$.

\section{CONCLUSION}

New accuracy factors (cosine function to the $n$th power) are proposed for incorporation into the NUFFT technique to improve the accuracy of the transformed data. The current density of a microstrip line is used for test of accuracy. Except for microstrip with offsets in a small region, minimal errors occur near or at $n=$ integer. It is suggested $n=4$ be used for transforms of symmetric input data and $3 \leq n \leq 4$ be tested for an optimal $n$ for asymmetric waves. When $n$ is an integer, the computation complexity is almost the same as that reported in previous literature. The NUFFT data show that the errors of proposed factors

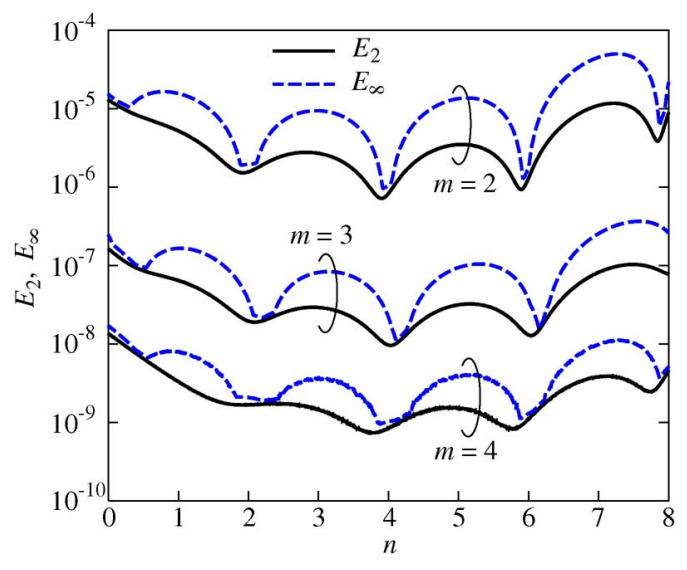

Fig. 4. Dependence of $E_{2}$ and $E_{\infty}$ errors on $m . q=8$ is used.

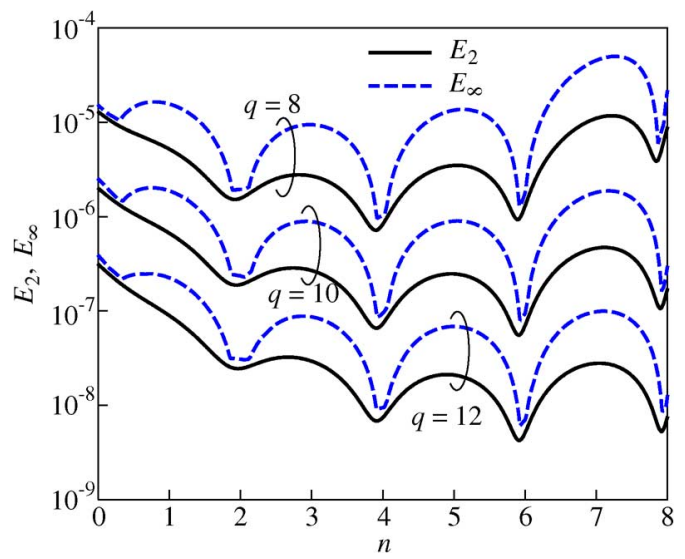

Fig. 5. Dependence of $E_{2}$ and $E_{\infty}$ errors on $q . m=2$ is used.

are only one third to less than one tenth of that of the existing cosine factors.

\section{REFERENCES}

[1] J. W. Cooley and J. W. Tukey, "Algorithm for the machine computation of complex Fourier series," Math. Comp., vol. 19, no. 90, pp. 297-301, 1965.

[2] A. Dutt and V. Rokhlin, "Fast Fourier transform for nonequispaced data," SIAM J. Sci. Comp., vol. 14, no. 6, pp. 1368-1393, 1993.

[3] Q. H. Liu and N. Nguyen, "An accurate algorithm for nonuniform fast Fourier transform (NUFFT)," IEEE Microw. Guided Wave Lett., vol. 8 , no. 1, pp. 18-20, Jan. 1998.

[4] N. Nguyen and Q. H. Liu, "The regular Fourier matrices and nonuniform fast Fourier transform," SIAM J. Sci. Comp., vol. 21, no. 1, pp. 283-293, 1999.

[5] K.-Y. Su and J.-T. Kuo, "An efficient analysis of shielded single and multiple coupled microstrip lines with the nonuniform fast Fourier transform (NUFFT) technique," IEEE Trans. Microw. Theory Tech., vol. 52, no. 1, pp. 90-96, Jan. 2004.

[6] K.-Y. Su and J.-T. Kuo, "Application of two-dimensional nonuniform fast Fourier transform (2-D NUFFT) technique to analysis of shielded microstrip circuits," IEEE Trans. Microw. Theory Tech., vol. 53, no. 3, pp. 993-999, Mar. 2005.

[7] I. S. Gradshteyn and I. M. Ryzhik, Tables of Integrals, Series, and Products. New York: Academic Press, 1980, p. 25. 